\title{
Decay Modes of Narrow Molecular Resonances
}

\author{
S. Courtin ${ }^{1}$, F. Haas ${ }^{1}$, M.-D. Salsac ${ }^{1}$, D. Lebhertz ${ }^{1}$, A. Michalon ${ }^{1}$, C. \\ Beck $^{1}$, M. Rousseau ${ }^{1}$, A. Sanchez I Zafra ${ }^{1}$, D.G. Jenkins ${ }^{2}$, R.G. Glover ${ }^{2}$, \\ P.E. Kent ${ }^{2}$, D. Hutcheon ${ }^{3}$, C. Davis ${ }^{3}$, J.E. Pearson ${ }^{3}$ and the Dragon \\ collaboration $^{3}, \mathrm{~K}$. Lister $^{4}$ \\ ${ }^{1}$ IPHC, UMR-7178, ULP(Strasbourg1) and CNRS/IN2P3,F-67037 Strasbourg Cedex 2, France \\ ${ }^{2}$ Department of Physics, University of York, Heslington, York YO10 5DD, United Kingdom \\ ${ }^{3}$ TRIUMF, 4004 Wesbrook Mall, Vancouver, BC, Canada \\ ${ }^{4}$ Physics Division, Argonne National Laboratory, Argonne, Illinois 60439, USA
}

\begin{abstract}
The heavy-ion radiative capture reactions ${ }^{12} \mathrm{C}\left({ }^{12} \mathrm{C}, \gamma\right){ }^{24} \mathrm{Mg}$ and ${ }^{12} \mathrm{C}\left({ }^{16} \mathrm{O}, \gamma\right)^{28} \mathrm{Si}$ have been performed on and off resonance at TRIUMF using the Dragon separator and its associated BGO array. The decay of the studied narrow resonances has been shown to proceed predominantly through quasi-bound doorway states which cluster and deformed configurations would have a large overlap with the entry resonance states.
\end{abstract}

Keywords: Resonances, Nuclear Molecules, Light Heavy-Ion Reactions.

PACS: 25.70.Ef, 25.70.Bc, 21.60.Gx.

\section{RESONANCES IN LIGHT HEAVY-ION REACTIONS}

A large number of resonant-like features have been observed in heavy-ion reactions involving compound systems of masses $<60$. This is well understood in terms of phase space arguments: strong resonant effects are observed when the number of open channels is small [1]. What nevertheless remains an open question, despite the wealth of experimental data, is the link between these resonances and a marked cluster structure in the composite system. A way to establish this link is to study the decay of these resonances and measure spins, parities, fragment and gamma decay widths. Moreover, molecular states are based on deformed structures and, in that sense, the search for direct gamma decay of the resonant structures should show specific signatures of transitions between deformed states. This is however an experimental challenge since the radiative partial widths are expected to be very small (typically $10^{-}$ ${ }^{4}$ to $10^{-6}$ ).

In this paper, we would like to address the question of the link between resonant structures and molecular states by measuring the decay of narrow $(\Gamma \sim 150 \mathrm{keV})$ molecular resonances, corresponding to a rather long lifetime $\left(\sim 4 \times 10^{-21} \mathrm{~s}\right)$ for the composite system. We shall focus on the ${ }^{12} \mathrm{C}+{ }^{12} \mathrm{C}$ and ${ }^{12} \mathrm{C}+{ }^{16} \mathrm{O}$ systems for which such types of resonances have already been observed some 25 years ago at bombarding energies close to the Coulomb barrier and at low spin ( 0 to 4$)$. We will discuss experimental results on the radiative decay of these low energy and spin resonances. 


\section{THE ${ }^{12} \mathrm{C}\left({ }^{12} \mathrm{C}, \gamma\right){ }^{24} \mathrm{MG}$ AND ${ }^{12} \mathrm{C}\left({ }^{16} \mathrm{O}, \gamma\right){ }^{28} \mathrm{SI}$ RADIATIVE CAPTURE REACTIONS}

\section{Radiative capture of light heavy ions}

Radiative capture is a nuclear reaction in which the projectile is completely absorbed by the target nucleus and the compound nucleus formed is only cooled by $\gamma$ ray emission. This process is a powerful tool to determine spectroscopic properties of nuclear states. The main challenge in using radiative capture arises from the low crosssections for this phenomenon. The electromagnetic force being weaker than the nuclear force, the decay of unbound nuclear states by particle emission can be $10^{3}$ to $10^{6}$ times more probable than their $\gamma$-decay.

The radiative capture of light particles is commonly studied for nuclear astrophysics purposes. Followed by $\beta$-decay, it is an important part of the nucleosynthesis of medium mass nuclei in novae and X-ray bursters. What heavy ions are concerned, it is much less explored experimentally: the Coulomb barriers are much higher and the competition with the predominant fusion-evaporation channels is very strong. A.M. Sandorfi has reviewed extensively the radiative capture of heavy-ions in 1985 [2]. Pioneering studies concerning the radiative capture of heavy-ions have been performed by Keller et al. at GSI in the $80^{\text {ies }}$, measuring a $40 \mathrm{pb}$ cross-section for the ${ }^{90} \mathrm{Zr}\left({ }^{90} \mathrm{Zr}, \gamma\right){ }^{180} \mathrm{Hg}$ reaction using the SHIP velocity filter [3] and more recently, Camera et al. have studied the ${ }^{89} \mathrm{Y}\left({ }^{90} \mathrm{Zr}, \gamma\right){ }^{179} \mathrm{Au}$ at Argonne National Laboratory [4]. What the radiative capture of light heavy-ions is concerned, two systems have been explored the most: ${ }^{12} \mathrm{C}+{ }^{12} \mathrm{C}$ and ${ }^{12} \mathrm{C}+{ }^{16} \mathrm{O}$. These studies have been made by Sandorfi, Nathan, Collins et al. at Brookhaven National Laboratory $[5,6]$ and it has been shown that the cross-sections for both systems exhibit narrow resonances. The resonance yields are of the order of 20-40 nb/sr and their widths are around 150-200 keV. The $\gamma$ cooling following the capture was measured with a large $\mathrm{NaI}(\mathrm{Tl})$ crystal surrounded by a plastic scintillator for the rejection of not fully absorbed $\gamma$-ray events. Only high energy $\gamma$ rays could be measured in these experiments because of a high background at low energy due to the piling-up of transitions coming from the dominant fusionevaporation channels in the scintillating crystal. This background would be substantially reduced with the use of a recoil spectrometer to detect the $\gamma$ rays in coincidence with the compound nucleus. In those pioneering studies, only decays of the resonances to the low lying states of the compound nuclei were observed and interpreted in terms of giant quadrupole or dipole resonances.

More recently, Jenkins have reopened the case of the resonant ${ }^{12} \mathrm{C}\left({ }^{12} \mathrm{C}, \gamma\right)^{24} \mathrm{Mg}$ reaction in a campaign of experiments [7]. They have performed a first experiment at Berkeley National Laboratory using the Gammasphere array as a calorimeter and showing that a substantial part of the decay of the resonances in ${ }^{12} \mathrm{C}\left({ }^{12} \mathrm{C}, \gamma\right){ }^{24} \mathrm{Mg}$ proceeds via the $\mathrm{K}^{\pi}=2^{+}$band in ${ }^{24} \mathrm{Mg}$ and also feeds previously unobserved high lying doorway states at $\mathrm{E}_{\mathrm{x}} \sim 10 \mathrm{MeV}$. In a second experiment, the total radiative capture cross-section for ${ }^{12} \mathrm{C}+{ }^{12} \mathrm{C}$ has been measured at Argonne National Laboratory using the Fragment Mass Analyser (FMA). It has been shown that, due to the new 
decay paths, the capture cross-section strongly exceeds the value reported by Sandorfi et al. [2,5]. The ${ }^{24} \mathrm{Mg} 10 \mathrm{MeV}$ excitation energy region is where shape isomers with a ${ }^{12} \mathrm{C}-{ }^{12} \mathrm{C}$ structure are predicted. Descouvemont and Baye have predicted ${ }^{12} \mathrm{C}+{ }^{12} \mathrm{C}$ cluster bands in the generator coordinate method framework [8] and $10 \mathrm{MeV}$ transitions could be transitions between low-lying members of these bands. The same type of cluster bands have been recently predicted by Ohkubo and Yamashita using a deep-potential developed to describe the 'rainbow scattering' in ${ }^{12} \mathrm{C}+{ }^{16} \mathrm{O}$ [9].

For these systems, we have performed two experiments at Triumf (Vancouver) to measure these transitions on and off resonance and evaluate the branching ratios to the doorway states in both systems taking advantadge of the Dragon spectrometer for the detection of the ${ }^{24} \mathrm{Mg}$ and ${ }^{28} \mathrm{Si}$ recoiling compound nuclei and its efficient associated $\mathrm{BGO}$ array for the detection of high-energy $\gamma$ transitions.

\section{The Triumf - Dragon Experiments}

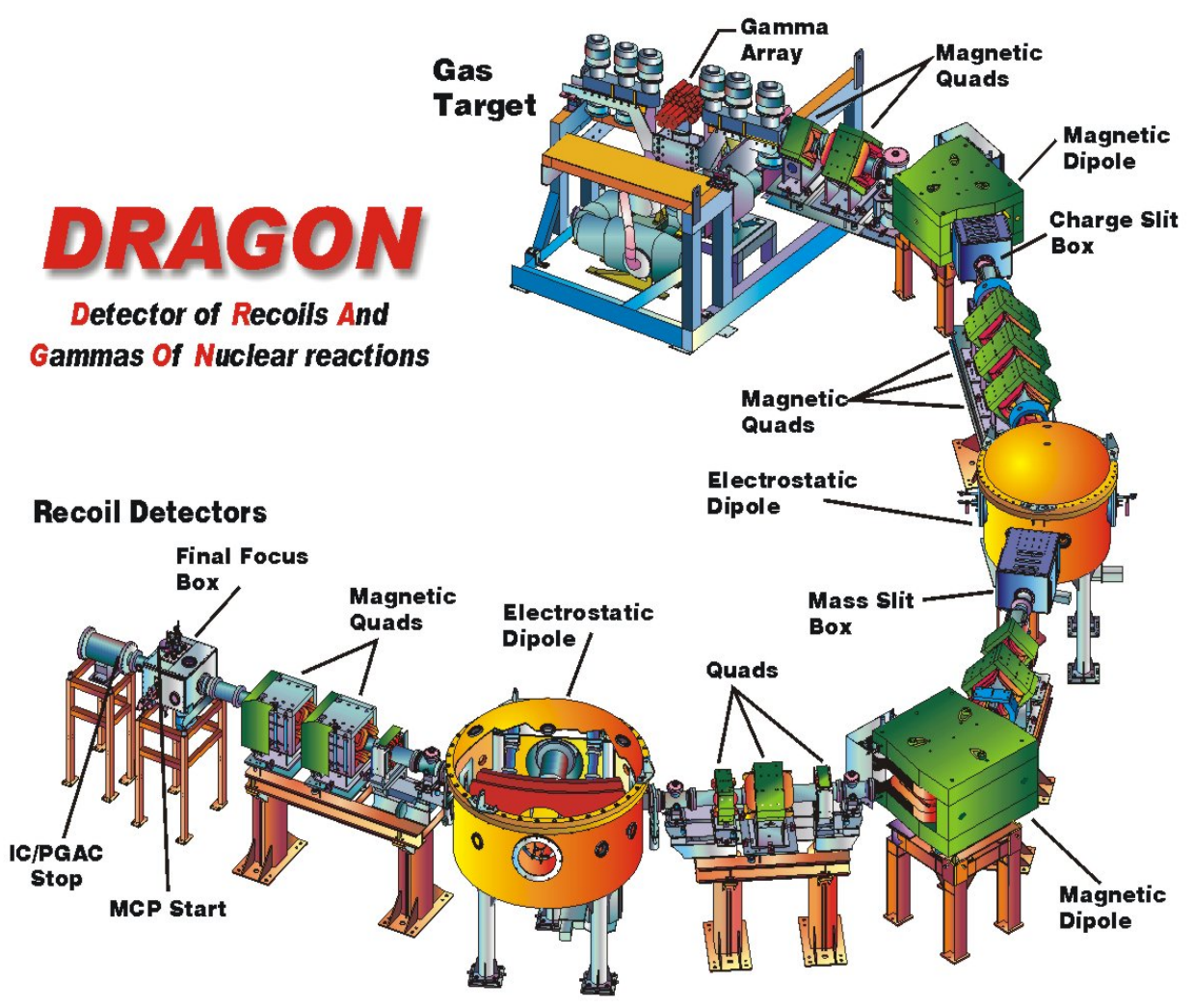

FIGURE 1. Layout of the Dragon spectrometer, from Ref. [10].

The Dragon spectrometer has been originally designed to study radiative capture experiments of astrophysics interest: typically $(p, \gamma)$ reactions, measured in inverse kinematics using a windowless gas target. In our experiments, this gas target has been replaced by a solid target ladder system to handle enriched ${ }^{12} \mathrm{C}\left(40 \mu \mathrm{g} . \mathrm{cm}^{-2}\right)$ targets. Measurements have been performed on and off resonance for both systems. Three resonances have been studied for ${ }^{12} \mathrm{C}\left({ }^{12} \mathrm{C}, \gamma\right){ }^{24} \mathrm{Mg}$ at $\mathrm{E}_{\mathrm{CM}}=6,8$ and $8.7 \mathrm{MeV}$ and two for the ${ }^{12} \mathrm{C}\left({ }^{16} \mathrm{O}, \gamma\right){ }^{28} \mathrm{Si}$ reaction at $\mathrm{E}_{\mathrm{CM}}=8.5$ and $9 \mathrm{MeV}$. The ${ }^{24} \mathrm{Mg}$ and ${ }^{28} \mathrm{Si}$ recoils 
have been selected in the Dragon spectrometer and detected at the focal plane in a double sided silicon strip detector (DSSSD). The Dragon $0^{\circ}$ spectrometer shown on Fig.1, based on two stages mass and charge separation, allows a very high rejection rate (typically $10^{13}$ ) of the incident beam particles which is of course essential to select the recoiling nuclei of interest. The $\gamma$ rays in coincidence with these recoils were detected in the associated $30 \mathrm{BGO}$ array covering $90 \%$ of $4 \pi$ and providing a high efficiency $\gamma$ detection $(50 \%$ at $5 \mathrm{MeV})$ but a rather poor energy resolution. Fig. 2 a. shows the recoils energy spectrum recorded in the DSSSD on resonance $\left(\mathrm{E}_{\mathrm{CM}}=8.5\right.$ $\mathrm{MeV}$ ) for the ${ }^{12} \mathrm{C}\left({ }^{16} \mathrm{O}, \gamma\right){ }^{28} \mathrm{Si}$ reaction with a $8 \mathrm{MeV}$ peak corresponding to the ${ }^{28} \mathrm{Si}$ recoils and a small peak at approximately $5.5 \mathrm{MeV}$ corresponding to a detector chamber $\alpha$ radioactivity. Fig. 2 b. shows the $\gamma$-spectrum in coincidence with the ${ }^{28} \mathrm{Si}$ recoils. This spectrum extends to $25 \mathrm{MeV}$, which is the excitation energy reached in ${ }^{28} \mathrm{Si}$ at the resonance energy. It shows strong transitions among the low-lying ${ }^{28} \mathrm{Si}$ excited states: $1.79 \mathrm{MeV}\left(2^{+} \rightarrow 0^{+}\right)$and $2.84 \mathrm{MeV}\left(4^{+} \rightarrow 2^{+}\right)$. The peaks observed around $4.5 \mathrm{MeV}$ and $6.9 \mathrm{MeV}$ probably correspond to the decays of ${ }^{28} \mathrm{Si}$ states at 6.28 $\mathrm{MeV}\left(3^{+}\right), 6.69 \mathrm{MeV}\left(0^{+}\right)$and at $6.88 \mathrm{MeV}\left(3^{-}\right)$. This spectrum of the $\gamma$ decay on resonance also displays a clear and broad structure centered around $13.5 \mathrm{MeV}$.

a.

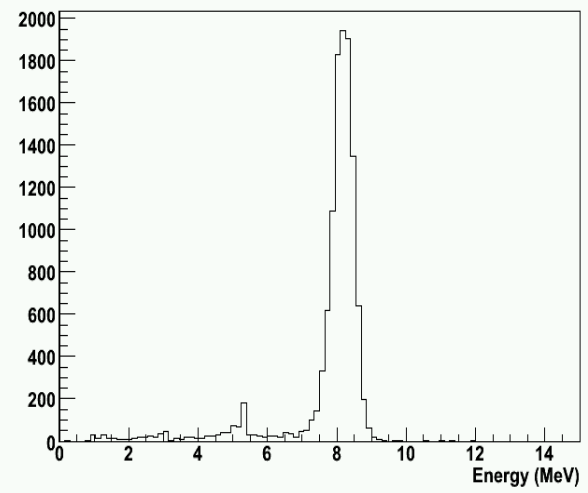

b.

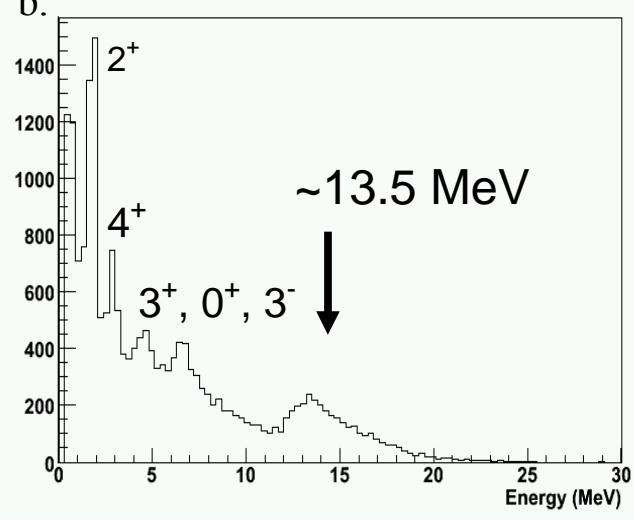

FIGURE 2. a. Recoils spectrum obtained on resonance in the ${ }^{12} \mathrm{C}\left({ }^{16} \mathrm{O}, \gamma\right)^{28} \mathrm{Si}$ reaction. b. BGO $\gamma$ spectrum in coincidence with the $8 \mathrm{MeV}^{28} \mathrm{Si}$ peak from spectrum a.

Fig. 3 displays spectra of the highest energetic $\gamma$-ray observed in coincidence with the recoiling heavy-ion obtained on resonance for the ${ }^{12} \mathrm{C}\left({ }^{16} \mathrm{O}, \gamma\right){ }^{28} \mathrm{Si}$ (Fig. 3 a.) and ${ }^{12} \mathrm{C}\left({ }^{12} \mathrm{C}, \gamma\right){ }^{24} \mathrm{Mg}$ (Fig. 3 b.) reactions at $\mathrm{E}_{\mathrm{CM}}=8.5$ and $6 \mathrm{MeV}$, respectively. Important structures are observed at $13.5 \mathrm{MeV}$ in ${ }^{12} \mathrm{C}+{ }^{16} \mathrm{O}$ and $10 \mathrm{MeV}$ in ${ }^{12} \mathrm{C}+{ }^{12} \mathrm{C}$, showing that doorway states around $12 \mathrm{MeV}$ in ${ }^{28} \mathrm{Si}$ and $10 \mathrm{MeV}$ in ${ }^{24} \mathrm{Mg}$ are strongly fed in the resonance decay for both systems. 
a.

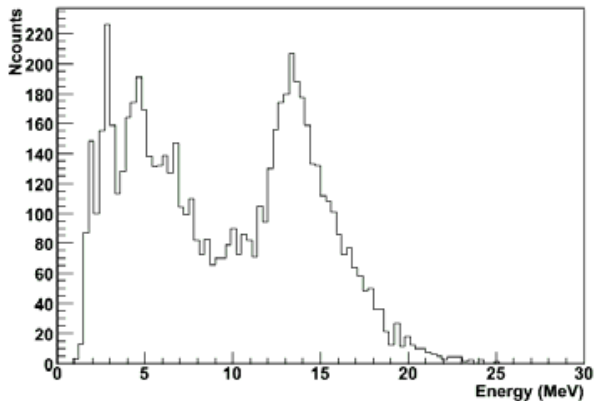

b.

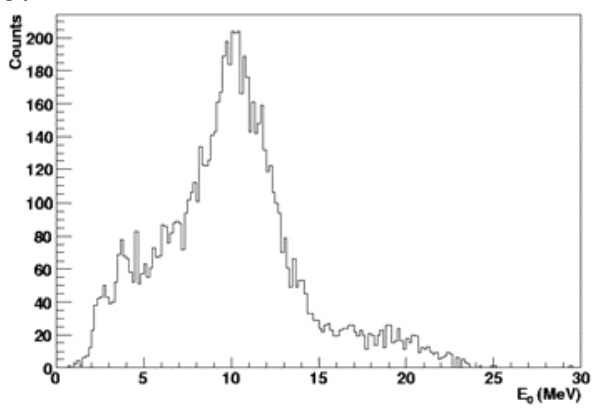

FIGURE 3. Highest energetic $\gamma$-ray spectrum observed on resonance in the ${ }^{12} \mathrm{C}\left({ }^{16} \mathrm{O}, \gamma\right)^{28} \mathrm{Si}$ reaction (a.) and the ${ }^{12} \mathrm{C}\left({ }^{12} \mathrm{C}, \gamma\right){ }^{24} \mathrm{Mg}$ reaction (b.).

\section{Discussion}

Both studies show that the decay of the resonances feeds predominantly states around $10 \mathrm{MeV}$ for the ${ }^{12} \mathrm{C}+{ }^{12} \mathrm{C}$ system and states around $12 \mathrm{MeV}$ for the ${ }^{12} \mathrm{C}+{ }^{16} \mathrm{O}$ system. Monte Carlo simulations using the GEANT 3 code have been started on the ${ }^{12} \mathrm{C}+{ }^{12} \mathrm{C}$ reaction to evaluate the branching ratio to those states compared to ground state or near ground state transitions. In a first step, calculations have been performed concerning the acceptance of the separator for recoiling ${ }^{24} \mathrm{Mg}$ nuclei emitting a cascade of two $\gamma$ rays of about $10 \mathrm{MeV}$ and those emitting a ground transition of 20 $\mathrm{MeV}$. The results are shown on Fig. 4.

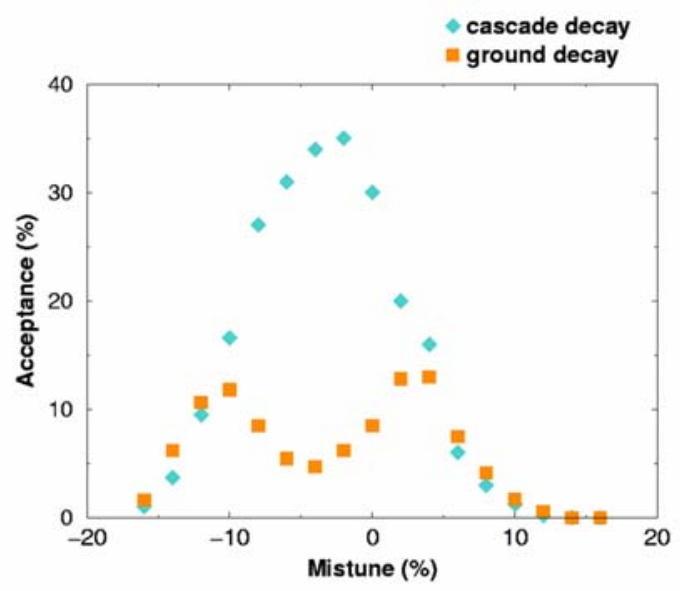

FIGURE 4. Geant simulation of the acceptance of Dragon as a function of the recoil tuning energy in the separator for a cascade decay of two $10 \mathrm{MeV} \gamma$ rays and a ground state decay for the ${ }^{12} \mathrm{C}\left({ }^{12} \mathrm{C}, \gamma\right)^{24} \mathrm{Mg}$ reaction.

The acceptance is clearly much larger for a cascade decay than a ground state decay. As Dragon has been built to study the radiative capture of light particles in inverse kinematics, its nominal acceptance is limited to a cone of half angle $20 \mathrm{mrad}$. Recoils may fall out of the separator acceptance in our experiments depending on the energy and angle of the $\gamma$ emission. The problem is of course more severe for recoils emitting large energy $\gamma$ rays, which is the case for ground state transitions. Monte- 
Carlo simulations involving low spin (0 or 2$){ }^{24} \mathrm{Mg}$ states around $10 \mathrm{MeV}$ are in progress. In both cases studied, the ${ }^{24} \mathrm{Mg}$ and ${ }^{28} \mathrm{Si}$ doorway states lie above the $\alpha$ emission threshold and thus the states involved are probably unbound with $\Gamma_{\gamma} / \Gamma_{\mathrm{T}} \sim 1$.

Differences can be observed in the BGO spectra shown in Fig. 3. The predominance of low energy $\gamma$ transitions in ${ }^{12} \mathrm{C}+{ }^{16} \mathrm{O}$ compared to ${ }^{12} \mathrm{C}+{ }^{12} \mathrm{C}$ is mostly due to a technical reason, the BGO ADC thresholds were indeed set at a higher level in the ${ }^{12} \mathrm{C}+{ }^{12} \mathrm{C}$ experiment. What the high energy parts of Fig. 3 spectra are concerned, the $\gamma$ transitions at energies higher than $\sim 20 \mathrm{MeV}$ are stronger in the ${ }^{12} \mathrm{C}\left({ }^{12} \mathrm{C}, \gamma\right){ }^{24} \mathrm{Mg}$ radiative capture. This may be due to two different reasons:

- the $\gamma$ transitions observed in the ${ }^{12} \mathrm{C}+{ }^{12} \mathrm{C}$ reaction are most likely to be of quadrupole nature, i.e. with angular distributions with maxima around $45^{\circ}$. In the case of ${ }^{12} \mathrm{C}+{ }^{16} \mathrm{O}$, dipole transitions may be observed with distributions maxima around $90^{\circ}$. For kinematical reasons, the corresponding ${ }^{28} \mathrm{Si}$ recoil angles may be larger than the separator acceptance and thus the corresponding high energy $\gamma$ rays would not be recorded in the coincidence spectrum;

- for both resonant radiative capture reactions, the entrance channel has a molecular-like prolate configuration. Such a deformed composite system has a large overlap with the prolate ground state band of ${ }^{24} \mathrm{Mg}$ but not with the oblate ground state band of ${ }^{28} \mathrm{Si}$. This could explain a larger feeding of the low lying ${ }^{24} \mathrm{Mg}$ states in the case of the ${ }^{12} \mathrm{C}+{ }^{12} \mathrm{C}$ reaction.

\section{CONCLUSION}

We have shown that the on resonance ${ }^{12} \mathrm{C}\left({ }^{12} \mathrm{C}, \gamma\right)^{24} \mathrm{Mg}$ and ${ }^{12} \mathrm{C}\left({ }^{16} \mathrm{O}, \gamma\right){ }^{28} \mathrm{Si}$ radiative capture reactions decay predominantly through ${ }^{24} \mathrm{Mg}$ and ${ }^{28} \mathrm{Si}$ doorway states at energies around $10 \mathrm{MeV}$ and $12 \mathrm{MeV}$ respectively. Monte-Carlo simulations are in progress to estimate the branching ratios of such transitions compared to the ground state or near ground state decays. In the case of ${ }^{12} \mathrm{C}+{ }^{12} \mathrm{C}$, a clear identification of the ${ }^{24} \mathrm{Mg}$ doorway states should come from our next experiment to be performed at Argonne National Laboratory in which we will take advantage of the large acceptance

of the FMA to select the ${ }^{24} \mathrm{Mg}$ recoils and identify the $\gamma$ rays in coincidence with the high resolution Gammasphere array.

\section{REFERENCES}

1. F. Haas and Y. Abe, Phys. Rev. Lett. 46, 1667 (1981).

2. A.M. Sandorfi, treatise on Heavy Ion Science, Vol.2, Sec. III (Ed. Allan Bromley) and references therein.

3. J.G. Keller et al., Phys. Rev. C 29, 1569 (1984).

4. F. Camera et al., Phys. Lett. B 560, 155 (2003).

5. A.M. Sandorfi and A.M. Nathan, Phys. Rev. Lett. 40, 1252 (1978) and A.M. Nathan, A.M. Sandorfi and T.J. Bowles, Phys. Rev. C 24, 932 (1981).

6. M.T. Collins et al., Phys. Rev. Lett. 49, 1553 (1982).

7. D.G. Jenkins et al., Phys. Rev. C 71, 041301(R) (2005).

8. P. Descouvemont and D. Baye, Phys. Lett. 169B, 143 (1986).

9. S. Ohkubo and K. Yamashita, Phys. Lett. B578, 304 (2004).

10. D.A. Hutcheon et al., Nucl. Instr. and Methods A498, 190 (2003). 